\title{
Review of Development of Multi-static Sonar for Underwater Object Detection
}

\author{
Yang Guang, Li Ye \\ Shandong Provincial Key Laboratory of Ocean \\ Environment Monitoring Technology \\ Shandong Academy of Sciences Institute of \\ Oceanographic Instrumentation \\ Qingdao, China \\ edit231@163.com
}

\author{
Xiang Xu, Wang Zhognqiu \\ Shandong Provincial Key Laboratory of Ocean \\ Environment Monitoring Technology \\ Shandong Academy of Sciences Institute of \\ Oceanographic Instrumentation \\ Qingdao, China
}

\begin{abstract}
In recent years, as the increasing requirement of submarine detection, the multi-static sonar technique is more and more investigated. Multi-static sonar has become one of the main techniques for countermeasure of silent submarine because of the advantages, such as large detection area, good occultation, flexible configuration, and strong ability of antidisturbance. In this paper, the state of the art of multi-static sonar research work is reviewed, and the representative positioning algorithms for multi-static sonar are summarized from multi-static sonar equation, that may be useful as reference of researchers.
\end{abstract}

Keywords-Multi-static sonar, Acoustic, Underwater object detection

\section{INTRODUCTION)}

As the development of noise reduction technology, the submarine becomes more and more silent, which means the acoustic emission from wall or screw is decreasing. It becomes more and more difficult for passive sonar to detect the submarine because the working distance for detection is shortened as the acoustic emission decreased. Especially when the marine stay stable, the passive sonar is completely useless. But the multi-static sonar is a powerful technique that can be used to compensate this shortage. Multi-static sonar is composed of several parts, including acoustic emission component, acoustic receiver distributed in different place in space. Acoustic emission component is sound source that emits sound power to space, the acoustic receiver is passive sonar that is used for collect the acoustic reflection from different places. Since the receivers stay static silently in any possible place, it is impossible for hostile to find out the exact position of receiver, as a result, it becomes difficult for hostile's countermeasure and avoidance. It is very adaptive for detection application of dipping sonar and joint detection application of submarine for vessel and aircraft's corporation because of the advantages, such as good occultation, strong ability of antidisturbance, easy realization and optimization, maneuvering and flexibility, long working distance, high positioning accuracy.

\section{StATE OF THE ARTS OF RESEARCH}

Detection and positioning of multi-static sonar could include several types in detail: single static sonar, bi-static sonar and multi-static sonar. Single static sonar is compromised of only one vector hydrophone to form a detection system, it is the basis of sonar detection. Bi-static sonar is the basic unit and most simplified form of compromising of multi-static sonar. Multi-static sonar is the expanded form and combination of bi-static sonar. The most famous research institutes and information platforms for multi-static sonar are listed below in Fig.1.
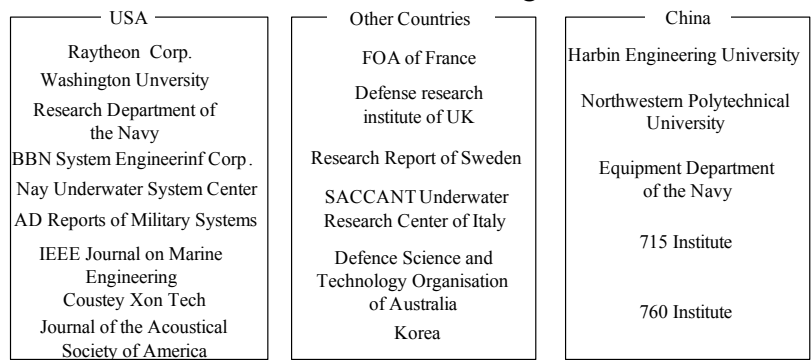

Figure 1. research institutes and information platforms for multi-static sonar

In USA, from 1950s to 1990 s, some research institutes and Research Department of the Navy, Raytheon Corp., Coustesy Xon Tech Corp., Nay Underwater System Center, Navy Underwater System Center, AD Report of Military Systems and Harvey conducted many research works about multi-static sonar, and series of results were obtained.

From 1990 to late 1990s, many other navy research institutes of other countries in the world also started similar works. The countries of NATO members conducted large amount of theoretical and experimental research works about static sonar detection, ocean bottom scattering, surface reverberation, forward-scattered direction properties of bistatic sonar. And meanwhile, many other development of multi-static sonar experiment system was conducted, which could be referred to the decrypted AD report and IEEE international conference papers. The detailed researches and reports are: naval dipping sonar experiments in 1992[1], the Washington University experiment in $1993[2,3]$, the FOA experiment of France in 1993[4,5], the multi-static active sonar system of dipping sonar buoy of Defense research institute of UK in 1994[6], "Research plan of multi-static sonar" of Sweden in 1995[7], expandable under water monitoring system experiment of SACLANT Underwater Research Center of Italy from 1995 to 1999[8]. In the research works above, the positioning and tracking properties of multi-static sonar were investigated, and the data fusion of multi-static sonar net was also researched preliminary. At the 
same time, the research of positioning precision of AUV (Autonomous Underwater Vehicle) under multi-static sonar configuration was also conducted.

In 1999, the paper of "overview of multi-static active sonar plan of Australia" is published in the fifth IEEE International Conference of Signal Processing and Applications [9]. In this paper, the research works about one type of multi-static sonar that was used was reported, and the properties of this system was introduced in brief.

In 2001, a paper about experimental measurement of the sea about high frequency and bi-static sonar bottom scattering was published $[10,11]$. In this paper, bi-static sonar experiment results were reported, conducted at the east coat of Korea by underwater research center of Defense Development Agency. On the international conference in 2002, authors of S Cho and J Chun from Korea published the paper "multi-static sonar positioning based on total least square method".

In 2002, a paper "the effect of sonar buoy position precision on positioning precision of multi-static sonar of dipping sonar" was published on Journal of Ocean Engineering of IEEE [12], which reflected the latest progress in this field in abroad countries. The Physics Laboratory of Washington University derived the model about "bi-static scattering model of high-frequency elastic seabed", and conducted the research about variation trend law of target intensity variation following the variation of target attitude angle and split point.

In 2006, there are two papers named "Vector intensity field scattered by a rigid prolate spheroid" and "Bi-static sonar applications of intensity processing" published by the Pennsylvania State University authors $[13,14]$. In these two papers, the acoustic intensity processing and application of bi-static sonar were referred from experiment and theory basis, and the method of underwater target judgment using the phase relation between pressure and particle velocity was proposed.

For China, the research of bi-static/multi-static sonar started from early 1990s. In 1991, the first paper of reviewing bi-static sonar research was published. In this paper, the properties of bi-static sonar and its key techniques for application were discussed. Later, during the 8th five years plan of China, Northwestern Polytechnical University, 715 Institute, 760 Institute etc. conducted some theoretical works about positioning theory, positioning methods, direction finding precision of bi-static sonar. In 2001, LING Guo-min reviewed the development of sonar technology in abroad countries, and reported the development and research trend of multi-static sonar. In 2002, the author HE Guozheng[15] of 715 institute reported the simple experiment on sea, and verified the possibility of multi-static sonar. At the same time, Northwestern Polytechnical University conducted the researches on localization and target property for bistatic/multi-static sonar[16]. And in 2006, the Equipment Department of the Navy and Harbin Engineering University conducted the study of detection of underwater object based on multi-static sonar system [17].

\section{THE SONAR EQUATION}

Under the noise constrained condition, the bi-static sonar equation could be described as Eq.(1) [18-20]

$S L-T L_{1}-T L_{2}+T S-N L+D I=D T$

Under the reverberation constrained condition, the bistatic sonar equation could be described as Eq.(2)

$S L-T L_{1}-T L_{2}+T S-R L=D T$

The two equations are referred to single basic sonar equation, where, $T L_{1}$ and $T L_{2}$ represent the transmission loss of sound travel from acoustic source to target and from target to receiver. The parameter $T L_{1}$ and $T L_{2}$ are generally different in bi-static sonar transportation conditions.

\section{REPRESENTATIVE RESEARCH METHODS}

The theory research of underwater target positioning, target tracking of single static, bi-static, multi-static sonar could be listed as the Fig.2.

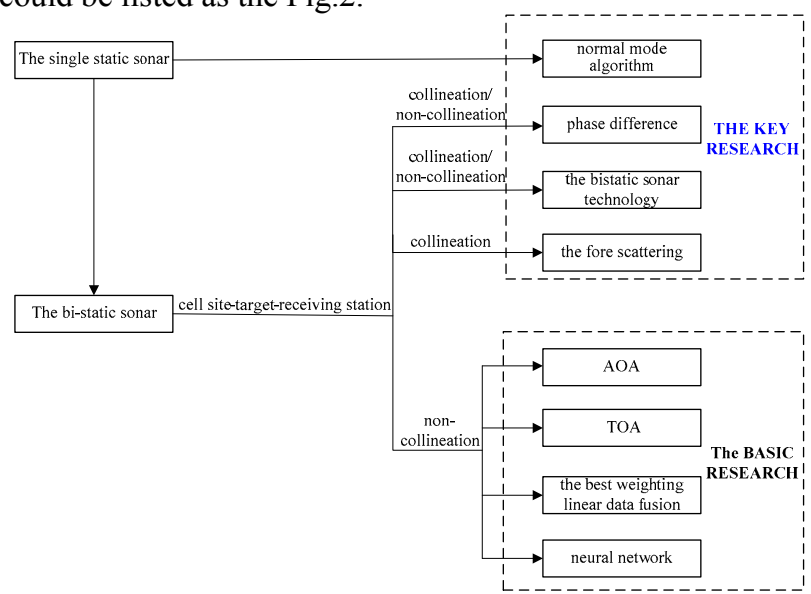

Figure 2. Representative methods of target detection, positioning and tracking

The bi-static sonar location can be achieved through DOA and TOA detecting and tracking positioning for the underwater target. And according to the time difference the direct wave and echo detection wave as well as the incident angle of echo wave, combined with the spatial geometry relationship of the three, the target can be explored. The algorithm principle is shown in Fig3 [21-23].

The separation of the surface targets and the underwater targets is realized through using the normal mode [24]. The algorithm principle is: according to pekeris shallow underwater acoustic model, the cross-spectral analysis is conducted, and taking advantage of the relationship of pressure and vertical velocity as well as the multi-order normal mode model, the active components are extracted and the distinction between surface targets and underwater targets is carried out. 


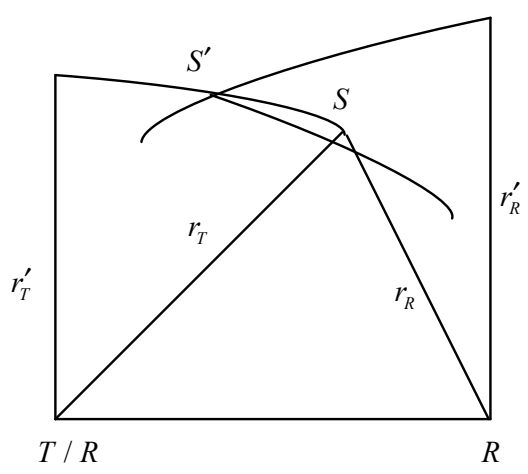

Figure 3. The principle of TOA and DOA algorithms

The target is not detected through pressure methods when the target is located in the connection of the two bases. Therefore, the phase difference of plural sound intensity is taken to detect the underwater target [13-14]. Or the detection way is changed, that is, the fore scattering algorithm is employed. The phase difference algorithm is adopted to detect target through the relationship of the pressure, the velocity and the target distance. The fore scattering algorithm, which solves the relationship of the sound energy and the distance of fore scattering, can successfully probe the target [25].

The neural network algorithm is used [26], according to the distance of multi-static sonar measurements and the nonlinearity location model, and the desired output matrix of network is got through the normalization of input matrix data of neural network, therefore, the target direction matrix is estimated. Furthermore, using input and output data, the basic function is trained, and the weight is fixed, and in turn the location is carried out. This algorithm is suitable to the different sonar distribution of nonlinear model of neural network, basic function construction, training methods, weight settings and so on [27].

\section{ACKNOWLEDGMENT}

This paper was supported by the National Natural Science Foundation of China under Grant 40806044, Supported by the Qingdao Science and Technology Planning Project Fund, China under Grant 10-3-4-9-2-jch and Supported by Research Fund for the Doctoral Program of the Shandong Academy of Sciences under Grant Y09-2.

\section{REFERENCES}

[1] Initial investigation into the methodologies to be used for helicopterbased bistatic sonar in shallow water, AD-A257865/6/HDM, 1992.

[2] Barrier search model using active bistatic sonar to protect a channel,AD-A264910/1/HDM,1993.

[3] Caruthers, J.W., Novarini, J.C. Modeling bistatic bottom scattering strength including a forward scatter lobe, IEEE Journal of Oceanic Engineering, Vol.18 No.2, April 1993, 100-107.

[4] Generic oceanographic array technologies (GOATs), 98-Bistatic seabed scattering measurements using autonomous underwater vehicles, ADA376854/XAB, 998.

[5] Mozzone, L. Bogi,S., Primo,F.. Deployable Underwater Surveillance System-analysis of experimental results Part ш, SR-288.La Spezia
Italy, NATO SACLANT Undersea Research Center,1998, ADA378131/XAB.

[6] Sullivan, S.F., Jr., Hursky, P, Measurement of sea bed topography and bistatic scattering coefficients with steered frequency source arrays, OCEANS'94. Oceans Engineering for Today, Technology and Tomorrow, Preservation Proceedings, Vol.3, 1994, 200-206.

[7] Submarine sonar system concepts for littoral waters, AD-A304 412/0/HDM, 1996.

[8] Mozzone, L., Bogi, S., Primo, F. Deployable Underwater Surveillance System-target localization with multiple sonar receivers,SR-317. La Spezia Italy, NATO SACLANT Undersea Research Center, 1998, ADA378091/XAB.

[9] Swift, M., Riley, J.L., Lourey, S., Booth, L. An overview of the multi-static sonar Program in Australia. Proceedings of the Fifth International Symposium on Signal Processing and Its Applications, 1999(ISSPA'99), Vol.1, 1999, 321-324.

[10] High Frequency bistatic scattering model for elastic seafloors, ADA373575/XAB, 2000.

[11] Pulse propagation and bistatic scattering, ADA396582/XAB, 2001.

[12] Michael Sandys-Wunsch and Mark G.Hazen, Multi-static localization error due to receiver positioning errors. IEEE Journal of Oceanic Engineering, Vol.27, No.2, April, 2002. 328-334.

[13] Rapids B R, Lauchle G C. Vector intensity field scattered by a rigid prolate spheroid $[\mathrm{J}]$. The Journal of the Acoustical Society of America. 2006, 120(1): 38-48.

[14] Naluai N K, Lauchle G C, Gabrielson T B, et al. Bi-static sonar applications of intensity processing. The Journal of the Acoustical Society of America [J]. 2007, 121(4): 1909.

[15] Huo guo-zheng. the experimental research of multi-static sonar. acoustics and Electronic Engineering (Supplement) [J]. 2002,4-7. (In Chinese)

[16] Zhang xiao-feng. the underwater target detection and positioning of $\mathrm{Bi}$ - Multi static sonar. Northwestern Polytechnical University Ph.D dissertation. 2003, 10 (In Chinese)

[17] Ling qing. The location technology research of underwater acoustic exploration based on Comprehensive information. Harbin Engineering University Ph.D dissertation, 2006, 7. (In Chinese)

[18] Li qi-hu. Introduction to Sonar Signal Processing, Beijing, China Ocean Press, 1985. (In Chinese)

[19] Xiao guo-you. The Processing and Application of Sonar Signal. Xi'an: Northwestern Polytechnical University Press, 1996. (In Chinese)

[20] Zhu dian. Active sonar detection information theory, Beijing: Ocean Press, 1990. (In Chinese)

[21] Mozzone, L,Bogi, S,Primo, F. Deployable Underwater Surveillance System-target localization with multiple sonar receivers,SR-317.La Spezia Italy, NATO SACLANT Undersea Research Center,1998,ADA378091/XAB.

[22] Gavish, M.Weiss, A.J. Performance analysis of bearing-only target location algorithms, IEEE Trans,AES-28(8), 1992, 817-828.

[23] He Lixing, Sun Zhong kang. Position location technique and GDOP analysis in multi-static systems, Proceedings of CIE1991, 47-51.

[24] Yu yun, Hui jun-ying, Chen yang. The target depth classification of low-frequency acoustic field in shallow sea . Physics. Vol.58, No.9, September, 2009.Page(s): 6335-6343. (In Chinese)

[25] Wang Shuozhong. Finite-difference time-domain approach to acoustic scattering problems. J. Acoust Soc, Am.99 (4), 1996, 19241931.

[26] Brian Bourgeois, Jeraid Caruthers. Neural network Parameter estimation for a bistatic scattering strength model, proceeding of the IEEE OCEANS'92, conference: 158-163.

[27] Mozzone,L, Bogi,S, Primo,F. Localization and fusion of echoes with deployable multi-static active sonar: evaluation of feasibility using experiment data. ECUA'98, Fourth European Conference on Underwater Acoustic, Roma, 1998. (In Chinese) 